\title{
EN LA CUERDA FLOJA. EL ACCESO NORMATIVO \\ EN PRACTICAL REALITY
}

\author{
Antonio Gaitán Torres \\ Departamento de Filosofía \\ Universidad de Granada \\ agaitan@ugr.es
}

RESUMEN: Este artículo presenta una alternativa a la dicotomía entre razones motivacionales y razones normativas. En Practical Reality $(P R)$, Dancy mantiene que ambas razones se identifican con estados externos al agente - "tesis de la identidad" (TI) - . Hay dos argumentos en $P R$ a favor de la TI: El primero se basa en una interpretación externista del significado de "tener una razón". El segundo presenta una vía indirecta, defendiendo que las adscripciones de razones según estados psicológicos internos no son casos centrales en las explicaciones normativas. Estas dos críticas ignorarían una fuerte intuición normativa: el "acceso epistémico". Si ésta es persuasiva, los estados internos no pueden ser eliminables.

PALABRAS CLAVE: normatividad, explicación, agencia, Dancy

SUMMARY: This paper presents an alternative account to the dichotomy between normative and motivating reasons. In Practical Reality (PR), Dancy argues that both types of reasons should be identified with external states of the agent — "identity thesis" (IT). There are two arguments in $P R$ for the IT: one is based on an externalist interpretation of expressions like " $A$ has a reason to $x$ ". The other is an indirect argument that claims that the ascription of reasons grounded on internal states cannot be central instances in normative explanations. Both arguments ignore a strong intuition: the "epistemic access". If this is a persuasive intuition, internal psychological states cannot be eliminated.

KEY WORDS: normativity, explanation, agency, Dancy

En este artículo criticaré la posición antipsicologista que Jonathan Dancy defiende en Practical Reality (en adelante, $P R$ ) frente a aquellas teorías que entienden los motivos del agente como estados psicológicos internos indispensables en la explicación de su conducta. Defenderé que la afirmación central de $P R$ - la "tesis de la identidad", según la cual el mismo tipo de consideraciones externas sirven para motivar la acción del agente y para juzgar la racionalidad de su acción - se opone de modo importante a: (i) ciertos rasgos estructurales bien asentados en nuestras explicaciones agenciales y (ii) a un importante sentido de normatividad, por el que nuestros estados psicológicos son susceptibles de ofrecer un nivel de justificación externo a su ocurrencia, sin que esto presuponga una referencia explícita a estados de cosas. 
El argumento externista de Dancy es susceptible de presentarse de dos formas. Por un lado, podemos centrarnos en qué teorías de la normatividad están implícitas en el debate entre quienes pretenden eliminar la referencia a estados internos y quienes niegan que ese movimiento sea posible. $P R$ serviría, en este nivel, como una guía avanzada dentro de este ámbito de discusión normativa. En este campo de batalla luchan actualmente externistas normativos extremos, que pretenden eliminar cualquier referencia a estados internos del agente, frente a quienes asignan un papel a esos estados. Éstos, a su vez, se dividen entre aquellos que entienden que el papel de los estados internos se reduciría al de meros componentes de una clase peculiar de hechos explicativos - externistas moderados o compatibilistas - y quienes consideran que esos estados internos son componentes constitutivos o necesarios en cualquier explicación agencial —internistas, subjetivistas o neohumeanos-.

No obstante, otra forma de defender la "tesis de la identidad" ha sido puesta de relieve recientemente por Dancy, en respuesta a algunas críticas. Esta clase de argumentos sustentan su fuerza en una razonable intuición argumentativa. Según ésta intuición, una tesis - la ineliminabilidad de los estados internos - no es defendible si instancia casos extremos, anormales o inusuales del fenómeno general que pretende explicar - la adscripción de motivos o razones motivacionales a un agente-. Dancy mantiene que defender que las creencias motivan al agente, o fundamentan su acción justificándola, es verdadero únicamente en situaciones muy peculiares. Esto lo lleva a concluir que los estados internos no pueden ser la norma general al adscribir las razones motivacionales. Tanto el primer modo de abogar por la eliminabilidad como esta segunda vía llegan a la conclusión de que ningún estado interno motiva la acción de un agente, ni sirve para justificar su racionalidad - en el sentido normativamente relevante por el cual pretendemos saber si las razones que tenía un sujeto para hacer lo que hizo eran adecuadas-.

Este artículo defiende una postura contraria, identificándose con un espacio dentro de la teoría de la normatividad que únicamente será esbozada de modo indirecto, al delinear las dudas que los ejemplos de Dancy despiertan. Un modo de criticarlos - y de negar la intuición normativa general que los soportan - trataría de aclarar la función que la noción de "acceso epistémico" cumple en algunas situaciones usadas por Dancy para apoyar su "tesis de la identidad". 


\section{Humeanos, neohumeanos y antihumeanos. Un breve repaso}

A grandes rasgos, $P R$ intentaría determinar el modo en que debemos entender las razones que motivan a un agente a realizar un acto, si éstas quieren ser respetuosas con el tipo de exigencias normativas características de cualquier explicación agencial. Dentro de esta exigencia por atender a la normatividad inherente a nuestras explicaciones agenciales, el libro de Dancy defendería la necesidad de variar el foco de explicación de nuestra conducta, foco que se mueve desde los estados internos de tipo psicológico hasta los estados externos, o los estados de cosas. PR trataría de aclarar, según esto, cuál es el modo correcto de entender la dimensión motivacional propia de la conducta del agente y su relación con la esfera externa o normativa.

Las explicaciones de la conducta se habrían entendido mayoritariamente, según la reconstrucción que $P R$ hace suya, como "racionalizaciones" que justificarían en un sentido mínimo la conducta, utilizando estados psicológicos internos con diverso contenido como elementos necesarios en la constitución de esas razones. Estos constituyentes internos se han conceptuado de forma variada, combinándose de diferentes modos y acentuando en grado diverso el peso de cada uno de ellos en nuestras explicaciones. En lo que la ortodoxia, no obstante, ha estado mayoritariamente de acuerdo es en destacar su ineliminabilidad en la explicación de la acción (Davidson 1963; Smith 1994, caps. 4 y 5; Millar 2002, p. 119).

La intuición que apuntalaría la oposición de $P R$ a este modelo es bastante directa. Si consideramos que un rasgo destacado de cualquier relación normativa es cómo se relaciona ésta con aspectos externos a su formulación, deberíamos aceptar - contra esa conocida imagen esbozada líneas atrás - que explicar normativamente la conducta de un agente requiere citar las consideraciones externas sobre las que éste actuó, y no sólo los estados internos que racionalizan su conducta (Baier 1985, p. 125; Smith 1994, p. 130).

Según esto, los estados internos del agente, en cualquiera de las variantes presentadas por el "psicologismo", no serían candidatos adecuados para la función de justificación propia de cualquier explicación, y por ello deberíamos oponernos a cualquier intento de entender las explicaciones agenciales en términos estrictamente psicológicos. En general, ha de ser conceptualmente posible que el mismo tipo de consideración externa motive al agente y pueda servir para justificar su acción. Podemos señalar, por lo tanto, que la oposición a esta familia de teorías en el ámbito de las razones motivacionales 
y la defensa derivada de un "externismo normativo" constituyen las dos tesis generales de $P R$.

Entre las tensiones obvias que cualquier lector de $P R$ percibe en relación con este proyecto, destaca la marcada oposición a la teoría de la motivación clásica contenida en sus páginas. Ni el esquema humeano ni la serie de derivaciones que éste ha acarreado diferencian tradicionalmente entre aquellas razones que justifican un acto y las que motivan o causan la realización de ese acto (Hume 1978, II.iii.l2, II.iii.3, III.i.2; Smith 1994, 2003, 2004 ha presentado un modelo humeano que sí destaca esta distinción).

Para esta familia de teorías, las razones que explican un acto y las que lo justifican convergen en los estados internos del agente. Esta intuición psicologista se alimenta de dos fuentes: por un lado se hace eco de una conocida tesis filosófica que niega, de inicio, cualquier normatividad en el dominio factual (Hume). Por otro lado, esta intuición es repetuosa con el propósito metodológico o explicativo general que subraya la necesidad de respetar un minimalismo ontológico en nuestras atribuciones de responsabilidad (Williams). Los dos tipos de razones necesariamente convergen en la primacía asignada a los estados internos del agente en nuestras explicaciones de su conducta. Tanto los estados cognitivos como los estados desiderativos pueden considerarse, según esta concepción, mecanismos normales en la explicación y la justificación de la acción.

Este modelo se ha caracterizado de diversas formas, si bien no todas igualmente críticas en cuanto al reconocimiento de sus límites y presupuestos. Atenderé a una formulación reciente, por el modo en que refleja el interés de este escrito y por la voluntad de ruptura que representa frente a una forma muy extendida de entender la teoría humeana. Según Michael Smith, podemos definir la teoría humeana de la acción de modo constitutivo: para que una acción pueda ocasionarse $-\mathrm{y}$, por consiguiente, ser susceptible de justificación- es necesario, sostiene esta interpretación, que el sujeto se encuentre en un estado interno orientado teleológicamente, es decir, en un estado desiderativo. Igualmente, podemos definir esta familia de teorías centrándonos en la forma en que la explicación de una acción requiere postular estados internos en el agente, sin pretender que ellos sean el origen de esa motivación ni que, en un sentido ulterior, tengan una función de justificación intrínseca.

Lo característico de los esquemas humeanos, según esta distinción, sería su pretensión general por hacer converger los dos tipos de afirmaciones en torno a ciertos componentes psicológicos. Esto 
equivaldría a defender que lo definitorio de una explicación humeana pasaría por postular la centralidad de ciertos estados internos - en su caso los deseos - en la constitución y explicación normativa de la conducta (Smith 1994, pp. 94-97, 130-131, introduce esta distinción y subraya la necesidad de entender en un sentido limitado las afirmaciones de Hume).

En relación con lo anterior, este artículo girará en torno a dos aspectos: en primer lugar propondré una lectura "divergente" de la relación entre los dos ámbitos. En un sentido diferente al defendido por Smith, mi interpretación de esta divergencia se concentra exclusivamente en el ámbito de las razones motivacionales, y en el modo en que ésta puede alumbrar cuestiones categoriales propias de ese ámbito - cuestiones fundamentalmente relacionadas con la referencia y el alcance de los enunciados en los que usamos el término "razón"-.

Es bastante común defender que los estados internos esenciales para la explicación de la conducta no son los estados que normativamente justifican esa acción, y que los dos ámbitos divergen a la hora de componer una teoría de la acción. Ser sensibles a esta divergencia equivale a negar que podamos identificar las buenas razones, interpretadas por muchos como estados de cosas externos, con las razones que explican la conducta, es decir, con los estados psicológicos que motivan a un agente.

La amplia receptividad hacia esa intuición sugiere tres posibles posturas en la teoría de la acción contemporánea. Como ya lo señalé, los humeanos han intentado una convergencia hacia esos componentes internos en todo el dominio normativo. Algunos autores neohumeanos aceptan la fractura entre esos dos ámbitos como forma de salvar lo que la teoría puede tener de intuitivamente aceptable en cada uno de esos contextos (Smith). Finalmente, algunos antihumeanos - entre los que se ubicaría Dancy - pretenden lograr una convergencia opuesta hacia los estados externos.

Defenderé, en primer lugar, que ni un sentido ni otro de esta convergencia respeta algunas de nuestras intuiciones sobre el modo en que actuamos y explicamos nuestra conducta. Tampoco hacen justicia a los diversos niveles en los que la normatividad está presente en una teoría de la acción sensible a la dualidad de alcance de nuestros enunciados normativos, y creo que esto es importante en el nivel teórico.

Para defender esto prestaré atención, en segundo lugar, a un fenómeno evidente en toda explicación, parcialmente ignorado en $P R$. Me refiero a la relación obvia entre la perspectiva desde la que se ofrece 
la explicación de una acción, la perspectiva particular desde la que se actúa y los diferentes sentidos en que usamos la expresión " $A$ está justificado para sentir/hacer/creer que $p$ ". Sugeriré que es posible interpretar estas locuciones haciendo referencia a una consideración que funciona como una razón externa, así como a un sentido que señala que tenemos que tener cierto estado en virtud de otros de nuestros estados internos.

Apuntes recientes de John Broome sobre los dos sentidos fundamentales mediante los que podemos asignar normatividad a un estado interno, así como algunas sugerencias de Robert Jay Wallace sobre las dos perspectivas típicas en nuestra explicación, hacen justicia a lo que Dancy deja intocado en $P R$. Ambas arrojan luz sobre el contenido normativo de aquellas expresiones en las que señalamos qué razón para actuar tiene un sujeto.

A continuación presentaré la tesis central de $P R$, que defiende la identidad entre razones motivacionales y razones normativas. Mencionaré previamente una distinción habitual que ayuda a entender cómo se articula lo propuesto por Dancy con la ortodoxia normativa.

\section{Razones motivacionales y razones normativas}

Dancy se ocupa de la forma correcta de entender las relaciones existentes entre los dos sentidos que atribuimos normalmente al término "razón". En formas oracionales similares a

\section{Juan hizo $\phi$ porque $\gamma$}

se mantiene la posibilidad de hacer inteligible la acción de Juan desde dos instancias contrapuestas. Estas instancias suelen presentarse de diferentes formas, y ya es habitual referirse a ellas como las dos perspectivas básicas desde las que explicamos una acción. Estos dos mecanismos explicativos nos permitirían entender las diferencias entre los dos contextos implícitos en (1). En este sentido, se suele apuntar la diferencia entre la "perspectiva intencional" de una acción y la "perspectiva deliberativa" como posible vía para captar una contraposición conceptual que haría evidente la diferencia existente entre esos contextos. No obstante, previo a esta distinción existe un procedimiento aún más general que permite hacer referencia al tipo de contenido que aislamos cuando formulamos dos clases de preguntas: aquellas que inquieren por los motivos psicológicos de la acción y las que remiten a aquello que justifica la acción (Anscombe 1963; Smith 1994, p. 95). 
Al explicar la acción de Juan, existe un sentido inmediato que apunta hacia el conjunto de estados internos que explican que éste actuara de cierta forma. Estos estados suelen incluirse en una serie de patrones teleológicos o causales de explicación. Denominamos a las consideraciones aisladas a partir de estas preguntas las "razones motivacionales" que Juan tiene para actuar. Entre estas razones encontraremos cualquier estado interno que sirva de respuesta a la pregunta "¿Por qué hizo Juan eso?" En la respuesta a esa cuestión se incluirían componentes tan variados como creencias, deseos y patrones disposicionales modelados a partir de esos estados internos.

Sin embargo, existe otro tipo de preguntas según las cuales - y mediante esquemas como (1) — nos referimos a la serie de requisitos normativos a los que está sujeta la acción de Juan. Estos requisitos hacen correcta esa acción, presentándola como aceptable dentro de un determinado sistema de normas, o en relación con la evidencia de que dispone el agente. Los diferentes sistemas normativos que nos permiten aislar este otro tipo de razones, así como los distintos criterios de éxito epistémico o prudencial que nos son propios, son tremendamente variados. Estos criterios normativos permean nuestra práctica cotidiana de forma esencial y nos obligan a utilizar ciertos requisitos de racionalidad evidencial, de prudencia a favor de la consecución de nuestros fines, o de moralidad, cuando juzgamos las razones que tiene un agente. Dos posturas destacan en $P R$ al discutir los contornos y la naturaleza de este ámbito normativo proyectado sobre nuestras actitudes intencionales. Los neohumeanos entienden que podemos responder a esta pregunta estableciendo una serie de constricciones sobre nuestros estados internos. Sin embargo, en el caso de Dancy, la interpretación que éste hace propia refiere al modo en que una razón en sentido normativo está conceptualmente ligada a los rasgos externos que justifican una acción. Después señalaré algunas cosas sobre esta interpretación del alcance del contexto normativo. Baste por ahora señalar que lo que Dancy defiende en ningún sentido es algo conceptualmente evidente (Smith 1994, p. 96; Dancy 2000, p. 2).

En relación con esta división, la tesis defendida en $P R$ mantiene que en ambos contextos - y a pesar de sus diferentes usos - se puede aislar un sentido central de "razón" (Dancy 2000, p. 6; Raz 2001). El sentido de razón privilegiado resultará evidente si mostramos el argumento general utilizado por los teóricos que entienden las razones que motivan como estados psicológicos internos y la posición de Dancy con respecto a este intento.

Según la ortodoxia: 
(1) Las acciones humanas son explicadas en términos de razones (Davidson 1963; Smith 1994, p. 116);

(2) Los estados agenciales - qua estados cognitivos internos- son necesarios para que las explicaciones en términos de razones sean operativas (Williams 1981);

luego,

(3) Las acciones deben ser necesariamente explicadas utilizando estados internos.

El contraargumento de Dancy no pretende ser incontrovertido, pero sí más plausible en lo que concierne a la normatividad de esas explicaciones.

Según Dancy:

(1) Las acciones humanas son explicadas en términos de razones;

(2) Las explicaciones en términos de razones son explicaciones normativas que relacionan consideraciones o rasgos externos y acciones del agente;

(3) Los estados agenciales — qua estados cognitivos internos- no pueden constituirse en razones en un sentido normativo;

luego,

(4) Las acciones deben ser explicadas apelando a estados normativos externos.

Obviamente, la premisa que se debe discutir es (2). Tradicionalmente esta premisa ha servido de base para los ataques realizados sobre el modelo humeano, amparados en la amplia y sonora acusación de que éste era poco sensible a ciertos rasgos normativos necesarios en toda explicación agencial (Nagel 1970, pp. 39-40, es la fuente clásica de esta crítica). Sin embargo, la forma en que estos ataques han entendido la normatividad a la que el esquema anterior hace referencia, relacionándola fundamentalmente con cuestiones prudenciales o morales, complica la posibilidad de equiparar el ataque de Dancy a estas variantes críticas y, en un sentido importante, deja intacta la intuición de que una teoría humeana es primordialmente una teoría sobre las razones motivacionales de un agente. Únicamente en un 
sentido derivado podríamos decir que se ocupa de cuestiones sustantivas (Smith 1994, p. 98). Para Dancy, por tanto, la manera en que debemos oponernos a las intuiciones humeanas pasaría por subrayar que la posibilidad de que las explicaciones agenciales sean normativas está conceptualmente ligada a rasgos externos de los componentes de esa explicación y no al contenido o carácter moral de un conjunto limitado de acciones.

Existen, como señalé al principio, dos grandes líneas argumentativas en $P R$ para plasmar la necesidad de que el significado de las razones en esos dos contextos apunte a estados de cosas susceptibles de ser el caso y no a componentes internos del sujeto. En la siguiente sección explicito esta "tesis de identidad". Después me ocupo de los dos argumentos presentados por Dancy a favor de esa tesis. Uno de estos argumentos se articula directamente, tras discutir el estatus de las creencias falsas en la explicación de la acción (Secc. 4). El segundo argumento es bastante curioso ya que - aceptando que sea posible que las explicaciones en términos de estados internos funcionen normativamente - Dancy sugiere, mediante un tipo peculiar de ejemplos, que la forma en que esas explicaciones son exitosas muestra su propia inviabilidad (Secc. 5). Finalmente expondré en qué sentido la "convergencia explicativa" entre buenas razones y razones explicativas no puede delinearse como $P R$ pretende (Secc. 6).

\section{Una lectura extrema del requisito normativo como vía contra el psicologismo}

Dancy considera que explicamos la acción de un agente cuando citamos la serie de consideraciones externas relevantes para el agente en el momento de su actuación (Dancy 2000, pp. 129 y 132). Dentro de esta concepción se incluye un requisito doble, relativo a las dos dimensiones citadas anteriormente: la dimensión motivacional y la normativa.

Un ejemplo conocido ayudará a ilustrar estos dos requisitos. Según la vieja historia, no siempre deberíamos suponer que lo que está ante nosotros es una botella de ginebra. ${ }^{l} \mathrm{Si}$ durante una calurosa tarde Juan tiene el deseo de beber algo fresco y cree que la botella que está ante él contiene ginebra, y según esta creencia bebe el contenido de ese vaso - cuando de hecho contiene amoniaco- debemos estar dispuestos a preguntarnos qué requisitos ha de tener su acción para que la consideremos sujeta a racionalidad o, si queremos, a evaluación normativa.

${ }^{1}$ El ejemplo es de Williams 1981. 
Un aspecto aceptado en esta historia, que me ayudará a introducir el primero de los requisitos citados, refiere al contenido de las creencias de Juan y al modo en que ellas determinan nuestras adscripciones sobre él. Suele convenirse que si Juan no sabe que lo que contiene ese vaso es amoniaco, y, además, no cuenta con los medios para averiguarlo, parece poco sensato acusarlo de actuar en contra de su juicio ponderado o, en un sentido más cotidiano, tacharlo de irracional. Puesto que las consideraciones que nosotros juzgamos relevantes para negarnos a beber de ese vaso no están disponibles para Juan (o no son accesibles desde su perspectiva) nuestra acusación — que no es más que una adscripción de razón sobre el agente- parece depender, en cierto sentido, de que éste disponga de esa información. ${ }^{2}$

Según esto, todas las atribuciones de racionalidad sobre los agentes están sujetas a un requisito que relaciona el conjunto de aspectos normativos, evidentes desde la perspectiva externa del evaluador, con los rasgos propios del conjunto motivacional del agente.

Podemos enunciarla como sigue:

Requisito explicativo. Toda consideración normativa que se incluye en las "atribuciones de razón" realizadas sobre un agente debe poder estar disponible como un elemento dentro del conjunto motivacional de éste. ${ }^{3}$

Además de esto, las atribuciones de racionalidad sobre agentes están sujetas a un requisito inverso. Sin embargo, éste no parecerá tan indiscutible como el anterior. En nuestro ejemplo, la motivación o el deseo de Juan ha de estar en una determinada relación con las consideraciones normativas relevantes en su situación. Para Dancy, sólo podemos citar como motivación —o como elemento dentro del conjunto motivacional o de los estados internos del agente- un elemento que puede estar entre las buenas razones que favorecen nuestra actuación, o entre las cosas que harían correcta esa actuación. Suponiendo, de nuevo, que son los rasgos externos los que fijan la normatividad de una explicación:

Requisito normativo. Toda consideración motivacional que es incluida en las "atribuciones de razón" realizadas sobre un agente ha de ser la clase de entidad que podría estar dentro de una

${ }^{2}$ Véanse dos interpretaciones divergentes en Scanlon 1999, p. 26, y Parfit 1984, pp. 119 y ss.

${ }^{3}$ La formulación más persuasiva sigue siendo la de Williams 1981. Para la versión que se privilegia aquí, véanse Dancy 1994, p. 13; Dancy 2000, p. 101; y Smith 2004. 
explicación que atiende a las consideraciones relevantes - y por tanto debe ser ella misma una consideración externa-. (Dancy 1994, p. 13; Dancy 2000, p. 103)

Es conveniente entender el matiz que establece esta segunda condición. Los estados internos no pueden formar parte de las explicaciones de la acción no porque no guarden una relación adecuada con la serie de consideraciones externas que sirven para justificar la corrección de esa acción - algo parcialmente recogido en la condición anterior, cuando entendemos que fija precisamente una relación de accesibilidad-, sino porque "no son la clase de entidades normativas que pueden contribuir a 'explicar' y 'justificar' una acción". En el caso de Dancy, estas consideraciones o entidades refieren a estados de cosas susceptibles de ser el caso. Para el psicologismo estas consideraciones son estados psicológicos internos (Dancy 2000, p. 107).

Defenderé que la interpretación de Dancy del requisito normativo es demasiado literal. Esto se manifiesta tanto en su posición ante aquellos casos en que los agentes actúan según una consideración que no es adecuada (o que se manifiesta como falsa), como en la presentación de un argumento indirecto basado en un ejemplo. A continuación me ocupo del primero de estos casos. Sin embargo, sería conveniente subrayar que ambas vías pretenden sustentar su afirmación de la identidad entre las buenas razones y las razones que motivan al agente. En la sección siguiente, por tanto, presentaré el argumento psicologista basado en las creencias erróneas, argumento que pretende ser utilizado por Dancy, a través de su recusación, para sustentar una lectura extrema del postulado normativo.

\section{Creencias falsas y mecanismos de identificación entre razones motivacionales y normativas}

La tesis de Dancy a favor de la identidad entre contextos y su relación con el estatus explicativo de las creencias falsas es central en $P R$. Aclarar el papel de las representaciones mentales erróneas en la explicación agencial ha servido tradicionalmente para que los modelos psicologistas se fortalezcan en su interés por defender que explicar la conducta requiere necesariamente hacer referencia a estados internos del agente.

El caso de Juan sigue siendo paradigmático a este respecto. Según la interpretación de Dancy en $P R$ (Dancy 2000, pp. 102 y 132-133), las atribuciones reflejadas en esquemas oracionales como (1) deben interpretarse de la siguiente manera: 
(2) La razón de Juan para hacer $\phi$ es que $p$,

donde $p$ refiere a una consideración susceptible de ser el caso. Sin embargo, cuando no es el caso que $p$ parece claro reformular esquemas similares a (2) del siguiente modo

(3) La razón de Juan para hacer $\phi$ era que Juan creía que $p$.

Más allá de esta aparente obviedad, lo que quiero poner de relieve es que el psicologismo apuntala esta aparente banalidad y logra un argumento persuasivo similar al que presento abajo a favor de la ineliminabilidad de los estados internos (Bittner 2001, pp. 111-118):

(i) Postulado de uniformidad. La verdad o falsedad de las creencias del agente no deben alterar la forma de nuestra explicación.

(ii) La explicación de la acción de un agente cuando éste tiene creencias falsas no refiere al contenido posible de un estado interno, sino - si la explicación pretende ser relevante - a que el agente cree que $p$, es decir, al mismo estado interno (si Juan cree - falsamente - que el vaso contiene ginebra, cuando de hecho contiene amoniaco, la explicación de su acción deberá referir a su creencia).

(iii) La actuación del sujeto es indiferente al valor de verdad efectivo de su creencia (Juan beberá del vaso con independencia de que su creencia sea verdadera o no lo sea).

(iv) En los dos casos implícitos en (iii) — cuando su creencia es verdadera o cuando es falsa-, este hecho no altera la forma en la que debemos explicar la acción del agente, y así se respeta (i).

(v) Luego, (a) cuando explicamos la acción de un agente, nuestra explicación deberá referir a estados internos de creencia, independientemente de su verdad o falsedad y (b) son los estados psicológicos del agente los que explican su acción (Juan bebe del vaso porque cree que hay ginebra, y nada más es relevante para explicar su conducta).

La postura de Dancy frente a argumentos que van de (i) a (v) pasa por oponerse a esta familia de derivaciones, negando la relevancia que el psicologismo otorga al postulado reflejado en (i). En el ejemplo presentado por Dancy en el capítulo 6, y que comentaré después, es fácil apreciar que algunos psicologistas sostienen que el agente 
debe estar en el mismo estado interno durante el lapso que va desde nuestra adscripción de una razón sobre bases externas, hasta que comprobamos que la consideración adscrita no es el caso. Puesto que ese estado interno es una creencia en las situaciones fallidas - y puesto que presuponemos continuidad, tal y como (i) reflejaconcluimos que esos estados internos son la base sobre la que ese agente actúa en todas las situaciones (Dancy 2000, pp. 105, 123-125; Bittner 2001, p. 111).

Dancy mantiene que postular modelos psicologistas sustentados en este requisito de uniformidad supone comprometerse, de inicio, con defender que es imposible realizar una acción según las consideraciones que hacen correcta esa acción. No obstante, precisamente esto es lo que parece que se está discutiendo en ciertas versiones del psicologismo, por lo que la acusación de Dancy me parece apresurada. En seguida explico por qué.

Todos estaríamos de acuerdo, en principio, en convenir que la evidencia es un aspecto relevante en la atribución de razones a un agente, sin negar que una interpretación excesivamente factual de este requisito normativo casa muy mal con fuertes intuiciones sobre casos en los que el hecho postulado como justificación es erróneo, sencillamente no se produce o, en un sentido más complejo, no está disponible para el sujeto. En relación con esto, creo que Dancy está parcialmente en lo correcto al preguntar qué relevancia debemos otorgar a los casos desviados - casos de creencia errónea- cuando fijamos el funcionamiento normal de un proceso que él entiende centrado en la justificación evidencial.

No obstante, su reticencia metodológica general contra argumentos como el presentado antes no le permite inferir una conclusión tan fuerte como la que se presenta en $P R$. Para Dancy, es fundamental oponerse a estas derivaciones porque ninguna consideración sobre los estados mentales del agente puede señalar una diferencia normativa relevante en nuestras formas explicativas. Parece que Dancy destaca aquí que el tipo de consideraciones normativas que son relevantes cuando explicamos la acción de un sujeto requieren que cuestionemos la diferencia entre representaciones ajustadas y fallidas del sujeto, pero no el modo en que sus estados internos se relacionan entre sí. De esta convicción, de tipo representacional o evidencial, no se sigue, creo, que una diferencia en esos estados mentales no pueda fijar alguna diferencia normativa importante, al menos en el tipo de explicación que facilitamos cuando queremos explicar las razones del agente. 
La extrañeza general que nos provoca la oposición entre los dos ámbitos anteriores - el que refiere a las consideraciones normativas y el que alude a las explicaciones - reside, en gran medida, en que solemos utilizar un concepto de "normatividad" diferente asociado a cada contexto. Borrar esa diferencia es un mérito de $P R$, en la medida en que este movimiento sirve al propósito de reducir los componentes de la esfera motivacional a consideraciones normativas externas. Para Dancy, está claro que un agente debe actuar sobre la evidencia que favorece su acción si quiere que su conducta esté "justificada". En este sentido, que denominaré normatividad, quizás demasiado vinculado a los procesos de justificación epistémica, la crítica de Dancy sobre postulados como (i) parece perfectamente sensata. Sin embargo, cuando explicamos la conducta de un sujeto nos servimos además de ciertos términos primitivos - habitualmente creencias y deseos - que ayudan tanto a hacer inteligible el modo de proceder del agente, como a expresar ciertas relaciones inherentemente normativas.

Entender la diferencia entre los dos sentidos de normatividad que estoy delineando requiere únicamente reparar en la peculiar lectura que asignamos a "creer que $p$ " en adscripciones normativas peculiares. Cuando señalamos, por ejemplo, que $A$ está en estos tres estados: ${ }^{4}$

(i) Juan cree que el mundo fue creado en seis días.

(ii) Juan cree que si el mundo fue creado en seis días, entonces el mundo fue creado en menos de una semana.

(iii) Juan cree que el mundo fue creado en menos de una semana.

¿Estamos únicamente refiriéndonos al modo en que sus estados representacionales son sensibles a la evidencia? ¿Acaso señalar que $A$ tiene razones para tener esas creencias supone afirmar que $A$ tiene acceso — dejando de lado, por ejemplo, el tipo de colegio que $A$ ha frecuentado - a un cuerpo de evidencia que implica que cuando realizamos la adscripción que va de (i) a (iii) constatamos únicamente las razones que ya tenía o que la evidencia le suministraba? Parece que la relación normativa que va de (i) a (iii) es genuinamente independiente de la evidencia y, por tanto, no está sustentada en ningún hecho puramente externo. Sin embargo, ¿qué tipo de relación reconocemos cuando decimos que Juan debería tener esa creencia?

${ }^{4}$ El ejemplo es utilizado en varias ocasiones por Broome. 
¿Qué fallo comete quien adscribe (i) y (ii) y no es capaz de entender o prever que o bien $A$ deja de creer en (i) o deberá abrazar una creencia cuyo contenido sea (iii)? Ejemplos similares a éstos señalan, como bien lo ha hecho notar Broome, que nuestras adscripciones de creencias están sujetas a un tipo de relación en virtud de su contenido. Parece poco sensato equiparar esta relación a las que son propias del ámbito de la justificación evidencial externa.

En este sentido estrecho, ¿̨sostiene Dancy que ningún cambio en el comportamiento de alguno de estos componentes, expresados a través de verbos de creencia y estados con contenido volitivo, tiene repercusiones normativas para la forma de nuestras adscripciones y explicaciones? Es obvio que ahora hemos desviado el foco de manera importante hacia la explicación. Parece claro que si aceptamos que este nivel entra en nuestra explicación debemos convenir igualmente, a la luz de casos como el anterior, que existen ciertos requisitos internos sobre esas adscripciones, requisitos relacionados con esos componentes y su funcionamiento normativo. Denominaré a este segundo sentido "normatividad 2 ". 5

${ }^{5}$ La forma en que, por ejemplo, una intención irracional o poco justificada puede, no obstante, requerir del agente una determinada conducta recoge con acierto la divergencia existente entre los dos sentidos de normatividad identificados. Señalar que únicamente en los casos en que la evidencia externa nos apoya estamos obligados a realizar una conducta o a mantener una creencia, o tenemos justificación para hacerlo, pasa por alto cómo esos mismos estados internos nos comprometen con ciertos requisitos, de nivel normativo. En este sentido, nuestras intenciones o nuestros estados intencionales están sujetos a requisitos fijados a través de sus contenidos y la forma en que éstos se implican. La relación que nos imponen es diferente de la que existe entre esos estados y la evidencia externa, relación que solemos encuadrar en la justificación o el apoyo evidencial que ésta facilita. Que un estado intencional requiera normativamente otro estado se resume en que seamos capaces de interpretar formas condicionales como

(1) Si Juan cree que $p$ entonces debe creer que $q$

cuando es el caso que

(2) Juan cree que $p$,

lo cual no implica que $R(q)$ o $J(q)$ —no se implica que Juan tenga una razón o esté justificado para creer que $q-$, sino,

(3) $\operatorname{Debe}_{J}$ (si cree que $p$, entonces creer que $q$ ).

En la actualidad, Broome (1999, 2001 y 2002) y Millar (2002, pp. 123-125) desarrollan este punto general. No obstante, ninguno de ellos menciona explícitamente la necesidad de contar con estados internos para hacer operativa su propuesta. A pesar de esto, Broome señala los dos niveles propios en todo el razonamiento y los diferentes requisitos que suponen. Ambos intentos me parecen una buena forma 
Dancy debería mantener que una multitud de fallos epistémicos racionalmente requeridos — normatividad $_{2}$ - no son relevantes para fijar el nivel según el cual nuestra conducta es correcta, o sensible a la serie de rasgos externos — normatividad 1 - Sin embargo, parece natural señalar en algunos casos de creencias falsas o carentes de contenido intencional, pero normativamente requeridas, que es la propia creencia de Juan, quizás junto a otros estados, la que podría explicar su conducta — más allá del posible estado de cosas sobre el que éste pretendía actuar-y que Dancy privilegia debido a su negativa a aceptar que existe un nivel normativo propio de los estados intencionales. ${ }^{6}$

Por lo tanto, la identidad no funciona en todos los contextos, al menos en lo que se refiere a la explicación de algunas conductas, oponiéndose a lo que Dancy defiende al centrar nuestra atención en un único sentido, que aquí recojo bajo el rótulo de "normatividad 1 ". Si Dancy quiere ser coherente con su postulado de identidad, ¿ha de mantener que una creencia nunca podría formar parte de la justificación? Puesto que los estados que pueden ser objeto de explicación normativa deben ser capaces de ser el caso - condición que estaría en un nivel diferente del conjunto de fallos en la representación que nor-

de comenzar a reparar tanto en los diferentes sentidos de normatividad como en la posibilidad de ir adelgazando sus exigencias en relación con la experiencia.

${ }^{6}$ La distinción a la que aludo no aparece como tal en $P R$. Tampoco tiene relación directa con la más general entre razones motivacionales y razones normativas. Mientras esta última relación alude a las dos preguntas básicas que nos solemos hacer cuando explicamos una acción — ¿por qué? y ¿en virtud de qué?-, la distinción a la que me refiero cae exclusivamente dentro del segundo ámbito. En mi opinión, los dos tipos de relaciones pueden servir para justificar. Puesto que la tesis que defiendo en este artículo exige negar que los estados internos son eliminables, un modo indirecto de alcanzar ese objetivo pasaría por hacer persuasiva la intuición de que los estados internos también son requeridos normativamente, y por lo tanto están sujetos a un nivel de normatividad propio que impide su reducción. Por la forma en que la presento en mi escrito, la postura de Dancy parecería poco menos que banal; sin embargo, como señalé en la introducción, los externistas pueden ser moderados o extremos y creo que Dancy pertenece al primer grupo. En este sentido, el realismo extremo de Dancy se manifiesta en expresiones como: "aquello que motiva a un agente es idéntico a aspectos de la situación" o "a como son las cosas" (Dancy 2003, pp. 423 y 425). Coincido, empero, con uno de los árbitros en que algunos fragmentos podrían desviarnos de esta interpretación. Dancy afirma, por ejemplo, que esas consideraciones normativas pueden entenderse como "rasgos de la situación del agente" (Dancy 2003, p. 425) y en este sentido se lo podría interpretar como un realista moderado que entiende que "hechos" podría referir a la relación entre los estados internos del agente y su situación. Ahora bien, el número de veces en que Dancy se pronuncia en estos términos es reducido en comparación con su estruendosa defensa de un externismo fuerte. 
malmente atribuimos al explicar creencias erróneas - parece que las creencias no serían componentes esenciales de los procesos de justificación propios del ámbito normativo.

Que Dancy es consciente de la fuerza de intuiciones contrarias a la "tesis de la identidad" lo demuestran las páginas que dedica a explicar la función de los estados mentales en nuestras explicaciones. Dancy sostiene la operatividad de los estados internos en los esquemas de atribución mediante dos movimientos. El primero extiende una distinción propia de la teoría de la explicación, y trata a los estados de creencia del agente como "condiciones que posibilitan" que las explicaciones normativas sean operativas, pero no como rasgos que en sí mismos favorezcan o justifiquen la verdad de esas explicaciones (Dancy 2000, pp. 127-128; Audi 2001, pp. 25, 28 y 30). ${ }^{7}$

El segundo mecanismo interpreta la referencia a las creencias en un sentido paratáctico, como comentarios sobre la serie de consideraciones que un agente tiene en cuenta a la hora de actuar y que constituyen sus razones. De este modo, (3) puede reformularse como

\section{(4) Juan hizo $\phi$ porque $p$, tal y como él creía,}

donde el comentario no especifica la razón del agente, sino que funciona como un comentario sobre esa razón, especificando uno de los rasgos relevantes para entender la conducta del agente (Dancy 2000, p. 129). ${ }^{8}$

${ }^{7}$ En la lectura radical del postulado de identidad, éste señala que lo que motiva a un agente a actuar es el estado de cosas creído, y no el estado de creencias que se le atribuye en la explicación. Ni siquiera el contenido de la creencia en un sentido clásico, es decir, entendido como un contenido proposicional, resulta un candidato adecuado para lo que Dancy considera posible de ser o no el caso. Cuando la creencia es falsa - en esta interpretación extrema del postulado-, estamos motivados por algo que no es el caso, y no por una creencia. (Véanse Dancy 2000, p. 115, y Scanlon 1999, p. 57, para una concepción cognitivista opuesta, en la que las entidades que pueden ser razones deben tener como contenido un estado de creencia expresado en términos proposicionales.)

${ }^{8}$ De nuevo, un aspecto importante de este tipo de esquemas - que queda claro en las reformulaciones paratácticas - es que el compromiso con que la normatividad de las explicaciones descanse en estados de cosas posibles no implica que éstos de hecho se produzcan. Por lo tanto, (4) es consistente con

(4*) Juan hizo $\phi$ porque $p$, tal y como él creía y $p$ es falso.

Un rasgo general de toda explicación normativa que refleja bien este segundo mecanismo es que cuando facilitamos explicaciones no nos comprometemos con que los estados de cosas que citamos dentro del dominio del comentario paratáctico sobre el agente ocurran de hecho, ya que nuestras explicaciones normativas no son de naturaleza factual, es decir, no exigen que lo que el agente crea deba producirse 
Las dos interpretaciones facilitadas por Dancy aceptan, sin embargo, que la viabilidad de cualquier solución que salvaguarde el estatus de las creencias en la explicación depende de una separación previa entre la perspectiva agencial y la perspectiva de quien explica la acción. Es bastante evidente en el primer mecanismo, por ejemplo, que el acceso a los rasgos externos que justifican una conducta es más directo para quien la explica que para quien la realiza. Sin embargo, Dancy no parece sensible a este hecho obvio como posible vía crítica a su propio postulado de identidad. Más adelante volveré sobre este punto, muy importante para lo que propongo aquí. Presento a continuación el argumento indirecto de Dancy a favor de la identidad.

\section{Pendiendo de una dudosa cuerda}

He presentado el argumento de índole general que Dancy formula a favor de la identificación de las razones motivacionales con estados de cosas externos. En sus últimos escritos sobre $P R$, Dancy ha insistido, sin embargo, en la importancia de un ejemplo concreto como vía indirecta para negar que las explicaciones agenciales en términos normativos requieran necesariamente postular estados internos sobre el agente (Dancy 2000, pp. 124-125; Dancy 2003, pp. 426 y 469). El ejemplo se presenta como un modo de oponerse a esquemas de sustitución ya familiares:

(1) La razón de $A$ para hacer $\phi$ era que $p$

syss

(2) $A$ creía que $p$,

luego,

(3) La razón de $A$ para hacer $\phi$ era efectivamente que $A$ creía que $p$

donde estos esquemas instancian argumentos que aprovechan la "tesis de la continuidad":

(i) Si un agente cree falsamente que $p$, entonces no podemos decir que su razón para actuar era $p$, sino "que él creía que $p$ ".

o ser el caso para que funcionen como explicaciones. Sólo requieren que estas explicaciones conserven, de algún modo, el compromiso epistémico del agente hacia esos estados de cosas supuestos o reales. Este segundo mecanismo recoge, en parte, este importante rasgo de las explicaciones (Dancy 2000, pp. 131-137). 
(ii) La distinción verdadero/ falso no es relevante para fijar la forma de nuestras explicaciones

(iii) luego, aun si la creencia fuera verdadera, la razón del agente debería ser "que él creía que $p$ ".

Dancy pretende mostrar que los casos en que el sujeto actúa según la descripción facilitada por (3) son inusuales en un sentido peculiar. Este sentido debería convencernos de que la forma adecuada de adscribir razones a un agente es la recogida en (1). Dancy mantiene que mientras los casos de creencias falsas son "el pan de cada día" para los agentes, los casos en los que esos agentes actúan tomando su creencia como una razón son mucho menos centrales que lo que (i) implica. Según esto, la continuidad deseada debe acometerse justo en sentido contrario al señalado por (iii).

Antes de presentar el ejemplo quiero mencionar que existe una presuposición en todo lo que sigue, la cual atañe a la relación existente entre casos anormales y casos centrales en la comprensión de un fenómeno. Según Dancy, los casos desviados deben entenderse como instancias de nuestra forma explicativa central, y quizás el modo en que esos casos son anormales requiera - antes que modificar nuestro esquema - preguntarnos por otras causas que pueden hacer que estos casos sean intratables en el modelo generalmente aceptado. Los casos que nos interesan son aquellos en los que un sujeto parece actuar de modo genuino conforme a su creencia y no conforme a un estado de cosas - como (1) sugiere - No obstante, es difícil enunciar de qué manera estos casos resultan extraños. Quizás un buen modo de explicarlo sea a través del mismo ejemplo.

Supongamos que estamos en la tesitura de descender un desfiladero. Justo antes de iniciar ese descenso, creemos que la cuerda que debe sostenernos está a punto de romperse. Con base en esta creencia, lo más normal es que nuestro nerviosismo suba unos enteros en la bolsa del miedo y decidamos que los deportes de riesgo corresponden a una edad que ya no es la nuestra. Este nerviosismo nos aconsejará que evitemos descender el desfiladero, incluso antes de saber si efectivamente la cuerda está o no en mal estado. Por tanto, y con independencia de que la cuerda efectivamente pueda romperse, la razón que motiva al agente para no escalar es su creencia de que la cuerda puede romperse, sea cual sea su verdad o correspondencia con cualquier estado de cosas. Podemos decir, en este caso, que el agente actúa conforme a sus creencias. 
El aspecto importante para entender este situación tiene que ver con las condiciones de individuación normativa. Defender que una forma adscriptiva similar a (3) —instanciada en este caso concretoes la razón por la cual el agente actúa supone contrastar sus razones con la serie de condiciones posibles en que las creencias que las sustentan persistirían. Mientras el agente crea que la cuerda está rota, su razón para no escalar deberá identificarse directamente con su creencia. En tales circunstancias, el agente tendría la misma razón si la cuerda no estuviera rota pero siguiera creyendo que pudiera estar en mal estado. Su creencia constituiría, en esta situación, una razón para no intentar escalar la montaña, con independencia de los hechos externos. La crítica de Dancy es que éste no es un caso normal ¿Por qué no lo es?

Para contestar este cuestionamiento es necesario preguntarnos por el modo en que estos ejemplos están sustentados en un esquema más general, que explicita cuál es la barrera que separa los casos normales de los casos desviados en la adscripción de razones. Este esquema refleja, en un sentido más general, el tipo de vínculo existente entre nuestras creencias y la base evidencial que las sustenta. Algo como lo presentado abajo subyace a estos ejemplos:

(1) Principio de operatividad representacional (P): que un estado representacional interno sea normativamente operativo para nuestra conducta en situaciones normales depende constitutivamente de su covariación respecto a las condiciones que lo harían verdadero (Dancy 2000, p. 124, y 2003, p. 426). ${ }^{9}$

(2) Principio de adscripción derivada: nuestras adscripciones normales sobre un agente derivan gran parte de su contenido de la operatividad de $(\mathrm{P})$, o del conocimiento compartido de que ciertos estados covarían junto a las condiciones que los hacen verdaderos.

(3) Casos límite: existen casos en los que un estado interno es operativo para nuestra conducta con independencia de las condiciones de covariación que le sirven de base, o en las que se sustenta normativamente.

${ }^{9}$ (P) implica, según Dancy, defender que cuando señalamos que una creencia es irracional nos estamos refiriendo a una situación en la que un agente podría estar sujeto a una razón con independencia de la covariación de su estado interno en relación con las circunstancias en que ese estado de creencia es verdadero. Una creencia racional, en cambio, covariaría con las condiciones de evidencia que la hacen verdadera. 
(4) Luego, los casos límite en que nuestras razones no covarían con la base de hechos implican — según (1) y (2) - que en esas situaciones no nos encontramos con un caso normal de adscripción.

Esquemas de sustitución en situaciones anormales similares a

(3) La razón de $A$ para hacer $\phi$ era efectivamente que $A$ creía que $p$

son correctos a condición de negar principios de gran fuerza intuitiva, como los apuntados en (1) y (2). Por consiguiente, el argumento de Dancy funciona como una reducción a partir de principios aceptados de modo mayoritario. Contra este tipo de esquema argumentativo, que apela a la solidez de principios independientes, quizás lo único que pueda oponerse sea una reformulación de alguno de esos principios en relación con el problema que nos ocupa.

Por lo tanto, criticaré este argumento cuestionando básicamente si la covariación entre creencias y evidencia se produce exactamente como (P) lo señala. Aceptaré para ello la intuición general implícita en $(\mathrm{P})$. Creo que nadie duda de que algo estructuralmente similar a este principio de soporte epistémico subyace en nuestras justificaciones normativas cotidianas. Sin embargo, no creo que la relación de covariación evidencial esquematizada arriba — relación bastante extendida en los modelos puramente epistémicos de justificaciónfuncione sin especificaciones ulteriores cuando pretendemos captar el tipo de relación normativa que se establece entre la posición del agente y la serie de consideraciones que favorecen su acción. En consecuencia, las razones normativas que un agente tiene no se dejan recoger totalmente en términos de covariación simple sobre una base evidencial.

En el ejemplo privilegiado por Dancy, sería muy precipitado señalar que el modo en que la creencia es ajena a la evidencia permite descalificar las formas de adscripción que privilegian estados internos. Esto resulta claro cuando reparamos en que la manera en que una creencia puede ser normativamente poco significativa para la conducta del sujeto no sólo depende directamente de la no covariación evidencial - como Dancy lo sugiere - sino también de la posibilidad de que el acceso que el sujeto tiene sobre esa posible covariación no resulte operativa para su conducta.

Una serie de puntualizaciones me parecen esenciales para que se entienda lo que quiero indicar con respecto a $(\mathrm{P})$ y a su relación 
con la cuestión del "acceso epistémico". ${ }^{10}$ En primer lugar no uso lañoción de "accesibilidad epistémica" como vía para salvar lo que la formulación de Dancy pueda tener de persuasiva; más bien, al contrario. Al formular esta noción en un sentido estrictamente normativo apunto a que los rasgos que son razones siempre son dependientes del acceso del agente - al facilitar éste el establecimiento de una vía de acceso a ellos-, pero en sí mismos nunca sirven como único fundamento sobre el cual establecer relaciones normativas entre una base evidencial, una creencia y su racionalidad.

En este sentido, sólo podemos postular con coherencia un principio como $(\mathrm{P})$ si el agente puede tener acceso al modo en que la evidencia varía. En el ejemplo de Dancy, únicamente señalaríamos que el agente mantiene una creencia normativamente poco significativa si, aun sabiendo que la cuerda no está en mal estado por cauces adecuados, él sigue creyendo que la cuerda podría estar rota. Si esto fuera así, creo que convendríamos que la mejor forma de captar lo que está pasando es señalar que la cuerda no está rota, ignorando la terquedad del asustado escalador basada en sus estados internos.

Pero nuestro ejemplo no apunta nada en relación con este dominio. ${ }^{11}$ En éste se pretende descalificar toda referencia a los estados internos del agente sin preguntar si puede acceder o no a las condiciones de acceso sobre las que solemos apuntalar la racionalidad de esos estados cognitivos y su posibilidad de covariación. Si modificamos (P) incluyendo una nueva condición de accesibilidad, el tercer paso de la derivación - la no variación de los contenidos internos y la posibilidad de actuar en algunas ocasiones sobre creencias - sería mucho más complicado de mantener. Esto es así porque estos casos caerían entonces del lado de la adscripción normal en situaciones en las que el agente no tiene acceso a esas condiciones de variación. El

${ }^{10}$ Goldman 1988 define esta noción y desarrolla la noción de transición requerida entre estados cognitivos.

${ }^{11}$ La intuición básica en la apelación a la accesibilidad es que las razón que un agente tiene para creer algo se compone a partir de tres niveles: (i) los rasgos externos materialmente accesibles al sujeto, (ii) aquellos rasgos de los que el sujeto tiene conciencia y (iii) los que figuran en una explicación adecuada. En este sentido, y como ha señalado Bittner 2001 (p. 124), los tres niveles son dependientes de cada sujeto de forma diferente. Mientras que dos sujetos pueden encontrarse en posiciones tales que los rasgos a los que acceden sean diferentes, se puede argüir que los estados cognitivos de cada agente fijan posteriormente diferencias en la relevancia dentro de ese dominio previo, dentro de ese espacio de razones. Finalmente, nuestra explicación selecciona también ciertos rasgos, y es más o menos correcta dependiendo de cómo lleve a cabo este proceso. Véase un argumento similar en Smith 2004. La postura de éste, no obstante, está en las antípodas del externismo de Bittner. 
argumento vía reductio sería, por lo tanto, más dudoso en caso de aceptar algo como lo siguiente:

$\left(\mathrm{P}^{*}\right)$ Principio de covariación modificado: las creencias y su operatividad para nuestra conducta dependen, en sentido constitutivo, (i) del modo en que éstas representan una situación y, en sentido necesario, (ii) del acceso efectivo del agente a las condiciones de variación de esa situación.

El ejemplo sustenta la reducción que Dancy pretende y funciona tomando como base una lectura extrema de $(\mathrm{P})$ que va contra aquello que intuitivamente aceptamos en $\left(\mathrm{P}^{*}\right)$. Sospecho que esta intuición ha sido parcialmente reconocida por Dancy en formulaciones anteriores (Dancy 1994, p. 4). Entendiendo de modo literal lo que Dancy señalaba entonces en torno al concepto de "razón", tanto que un agente haya realizado una acción "por una razón" como que podamos "explicar su acción según esa razón" implican que la razón estaba disponible, que él podía haber actuado teniendo en cuenta ese rasgo y que, en definitiva, cualquier justificación contendría este hecho obvio de manera necesaria. ${ }^{12}$

La postura general de Dancy ha variado tanto desde ese escrito, que la cuestión de la accesibilidad - que entonces merecía un tratamiento detallado fijando una importante condición sobre nuestras explicaciones - desaparece en $P R$ dejando como dos únicas máximas las que señalé en la sección 2 (Dancy 1994, p. 13).

A quienes esta serie de apuntes no los acabe de convencer les queda, a mi entender, la difícil tarea de explicarnos cómo cuestiones tan familiares como la que señala la "accesibilidad epistémica" pueden invocarse de modo inteligible sin incluir referencia alguna a los estados internos del agente. Estos estados fijan los contornos de acceso a los que estamos sujetos en cada adscripción externa. Igualmente, los verbos de creencia que los expresan fijan una serie de requisitos normativos en los límites que delinean esos contornos.

6. Algunas dudas generales sobre la tesis de la identidad:

la perspectiva deliberativa y su divergencia respecto a la explicativa

Concluiré afirmando que se puede esbozar una propuesta más razonable si se aceptan los tres vértices que $P R$ pretende oponer al favorecer una solución normativa extrema. Si tenemos en cuenta lo expuesto,

${ }^{12}$ Para un tratamiento detallado de la importancia de los estados de segundo orden en la definición de razón normativa se puede consultar Millar 2002. 
las dos facciones enfrentadas aceptan tres hechos fundamentales en toda explicación de la acción.

Parece claro (i) que tanto el psicologismo como los modelos realistas aceptan que atribuimos razones sobre la elección de un sujeto. Esto supone convenir que existen dos perspectivas básicas: la que refiere a la elección del agente y la de nuestra explicación de esa elección. $^{13}$

Parece aceptado por todos los frentes que (ii) - haciendo a un lado la validez de mi crítica a ejemplos como el presentado en la sección anterior - actuamos evaluando rasgos independientes de nuestra psicología. Esto equivale a señalar que lo que caracteriza, en un sentido interno o fenomenológico, a una elección es la evidencia que está presente para el agente en el momento de tomar una decisión (Dancy 2000, pp. 101, 103-105 y 107). De este modo, aquellos casos en los que un agente actúa sin considerar algún tipo de base evidencial - presente desde su perspectiva o accesible a él— pueden considerarse casos anormales o desviados de elección o, sencillamente, casos en los que no se elige de modo genuino.

Finalmente (iii), los estados de creencia del sujeto resultan en algún sentido indispensables en las atribuciones de razones que realizamos sobre cualquier agente que realiza una elección. Y - algo que no siempre se presenta como obvio - sobre el modelado de la misma situación de elección desde nuestra atribución externa.

Unir estos tres vértices de manera menos estruendosa pasaría por desarrollar la distinción entre diferentes sentidos de normatividad apuntada en este trabajo. Esta distinción es primaria, y llama la atención tanto hacia la complejidad que las adscripciones de racionalidad encierran como hacia los diferentes niveles de exigencia normativa que debemos tener en cuenta cuando las realizamos.

Una distinción estructural ha sido anotada adicionalmente por Robert Jay Wallace. Me refiero a la necesidad de subrayar la diferencia entre la perspectiva del agente en el momento de la elección y la

13 Hornsby 1997 (p. 283) hace un planteamiento similar, subrayando que los problemas clásicos sobre la agencia son embrollos conceptuales en los que nos preguntamos cómo relacionar dos perspectivas: por un lado, la perspectiva de primera persona, en la que un agente hace algo por una razón, y por otro, la perspectiva impersonal propia de la tercera persona, en la que tratamos de dar cuenta de la acción incluyéndola dentro de procesos causales externos. Este escrito comparte el deseo de anular esta oposición, pero no abraza ninguna teoría sustantiva acerca de cómo relacionar las dos perspectivas $-\mathrm{y}$ creo que tampoco se incluye en la senda clásica de la filosofía de la acción-. En el caso de Hornsby, cualquier explicación que señala las razones de un agente apunta hacia las causas de su acción. Para un tratamiento más detallado, véase Hornsby 1980, caps. 1-3. 
perspectiva de quien explica esa elección en un momento posterior (Wallace 2003).

En este trabajo he sugerido que la primera perspectiva hace uso del concepto de "razón motivacional" como Dancy pretende, mediante la identificación de los dos ámbitos básicos en los que nos referimos a la acción. En un sentido fenomenológico, desde la primera persona - $-\mathrm{y}$ en el momento de la elección - lo que cuenta como relevante son los rasgos externos, o las consideraciones relevantes, tal y como el agente las percibe. Desde esta perspectiva, primariamente agencial, no es relevante ninguna reflexión sobre nuestros estados internos (Darwall 1983, cap. 2; Scanlon 1999, p. 56; Millar 2002, p. 117).

Podemos, por tanto, interpretar el requisito normativo fijando una identidad en la primera persona entre lo que motiva y los rasgos que son percibidos como normativamente correctos. Esto haría natural interpretar cualquier razón, en el momento en que el agente actúa, como la clase de consideración susceptible de servir como una posible justificación para el sujeto. Esto supondría mantener de forma derivada que, en un imaginario informe personal durante un proceso de razonamiento, es poco probable que nos encontremos con formas como

(5) Yo hago $\phi$ porque creo que [creo que $p]$.

Contra esta falacia, debida a la equiparación o inclusión de la descripción externa de los procesos de elección dentro de la elección misma, la forma normal en que decidimos actuar suele depender del modo en que somos sensibles al contenido de determinados aspectos que se nos presentan como deseables — con independencia de los rasgos de nuestra propia perspectiva, o de las creencias que sirven de vehículo a nuestros procesos de razonamiento-. ${ }^{14}$

$\mathrm{Si}$, por lo tanto, el razonamiento es un proceso que tiene como materiales los diversos contenidos de nuestras creencias, entonces algo parecido a lo que Dancy considera el modelo de atribución general de razones parece la forma más normal de atribuírnoslas a nosotros mismos en el momento de actuar sobre esos procesos. Esquemas como el de abajo representan este hecho:

(6) Yo hago $\phi$ porque $p$.

${ }^{14}$ Bittner 2001, cap. 7, Wallace 2003, pp. 430-433, y Schueler 2003, pp. 58-60, exponen la falacia adscriptiva. Broome 2002, pp. 88-89, presenta un modelo de razonamiento práctico sensible a la diferencia entre el "contenido" del razonamiento y los "procesos" implicados en el mismo. 
Sin embargo, con la perspectiva externa pretendemos recoger la conducta del agente en un momento posterior a su actuación. Buscamos entonces fijar las consideraciones conforme a las cuales el agente actuó, sin hacer que la validez de éstas dependa de la psicología del sujeto. El ideal normativo sigue siendo el mismo y la variación de foco no afecta este deseo, como en algunos casos señala Wallace (2003, p. 432). Se trataría de indicar nuestro interés por hacer inteligible el punto de vista deliberativo. Por consiguiente, lo que estoy poniendo de relieve aquí es que tal vez ésta resulte una empresa impracticable sin mencionar estados internos de tipo psicológico. Hacer esto implica respetar el nivel de normatividad propio de estos componentes.

Wallace y yo tenemos dos puntos de acuerdo. Por un lado negamos que sea posible interpretar en sentido estricto el postulado normativo. Por otro sostenemos de forma derivada que los estados psicológicos no son eliminables ¿En qué difieren nuestras posturas con respecto a este último punto? Fundamentalmente nuestro desacuerdo reside en que Wallace se compromete con una postura realista que deja una función instrumental a los estados internos, contemplando un único tipo de relación normativa. En mi opinión, en cambio, ciertos estados internos se requieren mutuamente desde un punto de vista normativo. Mi propuesta y la de Wallace se diferencian, por lo tanto, en la relativa importancia que cada uno de nosotros otorga a los diferentes tipos de relaciones implicadas en esos dos modos de describir una acción. Simplemente Wallace reconoce un único tipo de relación donde yo he señalado dos. En consecuencia, Wallace otorga un papel normativo instrumental a los estados psicológicos. En mi lectura, estos estados internos tienen una función inherentemente normativa (Wallace 2003, p. 433; Dancy 2003, p. 470).

Si constatamos que existen dos sentidos en los que un estado puede justificarse, que esas dos relaciones puedan vehicularse desde una $\mathrm{u}$ otra perspectiva es, aunque interesante, secundario. Por ejemplo, en multitud de ocasiones se podría justificar una acción desde la primera persona por el modo en que algunas de nuestras acciones, deseos o creencias requieren, de nuestra parte, un determinado conjunto de estados internos. Tenemos sobrada experiencia de múltiples ocasiones en las que no actuamos considerando que tenemos una razón - entendida como una consideración externa que fundamenta nuestro estado - sino, más bien, "informamos" de que teníamos que sentir de tal modo, creer de tal otro, o actuar de tal forma. Y afirmamos, en un sentido un tanto pasivo, que no podíamos escapar a 
cierto nivel de exigencia que podemos justificar sin atender a razones externas. ${ }^{15}$

El hecho de que la dicotomía de Wallace deba supeditarse a los diferentes tipos de relaciones no supone que no podamos señalar qué tipo de relación es propia de cada perspectiva. En mi caso me interesa completar lo que dice Wallace añadiendo que ninguna explicación en tercera persona puede limitarse a describir los estados internos del agente dejando al margen la posibilidad de que algunos de esos estados guarden ciertas relaciones entre sí — del tipo simple por el cual $a$ requiere $b, b$ es requerido por $a$, o $a$ está en contradicción con $b$-.

Si esto es plausible, este trabajo defiende una "teoría híbrida" en el ámbito de la motivación, de un sentido común bastante evidente. Según esta teoría, los componentes internos no son eliminables de las explicaciones de la acción. Además, estos componentes no fijan un ámbito de justificación externo a esas conductas en situaciones normales. Es posible mantener estas dos afirmaciones sin renunciar a defender que esos estados son necesarios para considerar qué perspectiva ocupa el agente en relación con la evidencia que le facilita razones para su conducta. En ningún caso, a pesar de la debilidad de la formulación, los estados internos reflejan una normatividad que está fuera del agente. Por lo tanto, tener una razón en una situación es una función de la evidencia a favor de esa razón y de las posibilidades de acceso de un agente. Estas posibilidades se vehiculan a través de verbos psicológicos normativamente no eliminables.

${ }^{15}$ La distinción entre los diferentes niveles de normatividad usados en este trabajo no ha sido formulada por Wallace de manera notoria. Entre quienes de alguna manera han notado esta distinción se encuentran destacadamente Broome, Bernard Gert, Joshua Gert, Joseph Raz y Thomas Scanlon. Sólo los tres primeros le asignan un papel fundamental. No obstante, sería objeto de un escrito más largo examinar si esta distinción se podría solapar estrictamente con la distinción explicativa entre perspectivas planteada por Wallace. Mi intuición en relación con la primera persona es que podríamos atender a rasgos normativos en primera persona - como Wallace lo señala- porque otros estados requieren que consideremos un rasgo como una razón para actuar. Si esto es así - y los casos de debilidad de la voluntad serían un buen campo de trabajo como se sugiere indirectamente en la nota 5-, las relaciones normativas y sus características serían conceptualmente primarias para las diferentes perspectivas mediante las cuales explicamos la acción. Una segunda intuición implícita en la perspectiva de tercera persona señalaría — conectándola con las relaciones normativas primarias- que explicar desde esta perspectiva externa no sólo es señalar qué deseos y creencias racionalizan la acción, sino expresar, mediante esos estados internos, el tipo de requisito al que el agente está sujeto. Agradezco las sugerencias de uno de los árbitros en relación con este punto. 
Considero que Dancy está desencaminado en la crítica a esta serie de propuestas. Según él, todos los intentos de mantener que los componentes internos están normativamente cargados se sustentan, en última instancia, en un hecho que expresaría una relación entre esos estados - o entre esos estados y la evidencia-. Estos intentos tratarían de acercarse sin éxito a lo que su teoría logra sin ese "carácter híbrido". Además, esta "nueva teoría" no explica en qué consiste ese tipo de relación entre estados internos y componentes externos. En un sentido más grueso, además, ignora que las razones internas que nos motivan según esta nueva teoría no pueden ser la clase de entidades que pueden ser buenas razones (Dancy 2000, p. 119).

Esquemas como el siguiente $S$-s por una buena razón syss

(i) Hay una buena razón de $S$ para $\phi$

(ii) $S$ cree que hay una buena razón para $\phi$

(iii) $S$ hace $\phi$ porque él lo cree (Dancy 2003, p. 470)

explican las razones de un agente para actuar de una determinada forma a condición de que los estados internos del agente representen los estados de cosas que previamente son buenas razones. De este modo, la identificación entre las buenas razones y las razones que motivan se logra en estos esquemas psicologistas apelando a la mediación de la representación, e ignorando que esos estados internos ni pueden suscribir la serie de compromisos que Dancy señala como propios de los estados normativos, ni pueden captar - en cuanto (iii) debe entenderse conteniendo una especificación causal- el tipo de relación normativa propia de las razones y su relación con el agente - piénsese en las razones que son contrapesadas para entender que el vínculo causal no es el modo propio de entender estas entidades- (Dancy 2000, pp. 118119).

En consecuencia, el problema de Dancy es suponer que no podemos pretender ofrecer una genuina explicación de la acción, ser conscientes de la falacia adscriptiva y negarnos a defender una identidad entre razones motivacionales y normativas. Podemos hacer los dos primeros movimientos sin realizar el tercero, siempre que seamos conscientes de la normatividad que nuestros términos psicológicos encierran. En este sentido, las formas de atribución externas que 
favorezco serían equivalentes a enunciados sobre estados psicológicos estructuralmente similares a

(7) La razón de Juan para hacer $\phi$ es que él creía en $e$ que $p$

que tratarían de captar cómo ciertos rasgos se convierten en razones para el agente. ${ }^{16}$ Sólo serían razones motivacionales por el modo en que recogen —usando la serie de estados internos tradicionales - las consideraciones sobre las que el agente decidió tomar una decisión. No se limitan meramente a reflejar — como parece señalar Dancylos estados internos como tales, es decir, como estados psicológicos, sino que pretenden expresar una relación entre la evidencia externa y el acceso que el agente tiene a esa evidencia que es la base de la justificación.

En el caso de la reformulación del ejemplo presentado anteriormente, resulta evidente que existe cierta normatividad en la condición de accesibilidad, normatividad de una clase diferente de la postulación de una correspondencia con estados de cosas y demás entidades internas. Debido a la importancia de esta serie de elementos normativos estructurales en nuestros procesos decisionales - como la coherencia y la sensibilidad al cambio efectivo de los estados de cosas-, los estados internos pueden ser considerados estados susceptibles de ser verdaderos o falsos, ya que podemos reformularlos como

(7*) Juan tiene una razón motivacional adecuada para hacer $\phi$ syss (i) [que él creía que $p$ ] puede formularse según ciertos criterios y (ii) la razón de Juan para hacer $\phi$ es que $p$

donde

$\left(7^{* *}\right)$ [que él creía que $p$ ]

funciona como un hecho explicativo, es decir, como una entidad que respeta el requisito normativo señalado en la segunda sección, ya que

${ }^{16}$ Creo que esta condición es la que el mismo Dancy señalaba en 1994, cuando apuntaba que la elección entre creencias — entendidas como estados psicológicosy entidades normativas - entendidas como estados de cosas o hechos - desdibuja la existencia de una tercera opción: lo que motiva a un agente para actuar, y lo que justifica su acción en aquellos casos normativamente exitosos es lo que es creído por ese agente, no el estado de creencia en el que está. La forma en que Dancy definía entonces el papel de la creencia no es ajena al intento ecuménico de este escrito. Tampoco los son las puntualizaciones al proyecto presente centrado en la identidad. 
ni refiere meramente, ni representa de forma directa, estados internos del agente. Aunque sí requiere estados internos para expresar la relación normativa que está implícita. De este modo, $\left(7^{*}\right)$ y $\left(7^{* *}\right)$ expresan, en ejemplos como los favorecidos por Dancy, un hecho sobre los estados psicológicos de un sujeto, su relación con la evidencia y las posibilidades de acceso del agente a ésta. Algo bastante diferente de que ese contenido interno al que referimos mediante la jaula de corchetes pueda ser, por sí mismo, una buena razón para que un sujeto crea algo o actúe en virtud de ese contenido (Scanlon 1999, p. 57 , hace un señalamiento similar).

Igualmente, que $\left(7^{* *}\right)$ sea un hecho explicativo complejo hace que la crítica que subyace en gran parte de $P R$, que subraya la diferencia categorial entre las dos perspectivas, no afecte a esta solución. El compromiso epistémico del agente en primera persona puede mantenerse sin obviar la existencia de un hecho explicativo, formulado en tercera persona. Estas dos perspectivas no coinciden ni en el propósito ni en los componentes ni, obviamente, en su misma posibilidad de expresión. Como notablemente lo ha puesto de manifiesto Smith, tampoco coinciden en su instanciación sobre un mismo agente. Únicamente si existe previamente racionalidad en sentido sustantivo dentro del ámbito agencial, podemos esperar que nuestra reconstrucción normativa externa corresponda con un proceso decisional adecuado. Este ideal de racionalidad y su contingencia es la que explica que, a pesar de esa divergencia general indicada, nos sea posible con asiduidad explicar racionalmente la conducta de un sujeto (Smith 1994, p. 180).

Así, es muy plausible mantener que el compromiso agencial no debe demostrarse más que en las acciones del agente. Igualmente, lo único que nos interesa cuando explicamos la acción es reflejar el foco de la elección de la forma más fiable. Ambas perspectivas apuntan, por tanto, a niveles de normatividad distintos.

\section{Conclusión}

He tratado de defender un modo alternativo de entender la esfera motivacional y su relación con la normatividad que asignamos a nuestras explicaciones. En un sentido significativo, gran parte de lo que mantengo entra en la categoría de obviedad. Debido a que la principal línea argumental de $P R$ se apoya en la fuerza de algunas verdades supuestamente aceptadas por todos, quizá la mejor forma de oponerse a ellas sea apuntalar otras obviedades, confiando en que la partida no esté acabada con el movimiento que $P R$ representa. 
Para esto he abogado por una comprensión dual de las razones motivacionales. En lo que he presentado, el ámbito motivacional, que funcionaría de manera análoga a las consideraciones externas, y que nos motivaría a actuar en consonancia con rasgos externos, es el ámbito reducido de la deliberación en primera persona. Las razones motivacionales, sin embargo, tienen un rasgo normativo adicional asociado a una perspectiva diferente. Dancy parece ignorar esta perspectiva, asociada a una relación normativa no explotada en demasía por los teóricos de la normatividad. Las razones motivacionales cubrirían, según esta intuición, aquellas adscripciones sobre el agente realizadas por un evaluador mediante las cuales reconstruimos, usando estados internos, ciertos requerimientos objetivos sobre los actuamos de modo justificado. Estos estados normativos implican compromisos diferentes de los que Dancy supone propios a la relación de correspondencia con un estado de cosas. En un sentido importante, estas relaciones normativas hacen ineliminables las adscripciones de estados internos sobre el agente cuando explicamos su racionalidad.

Reparar en que algunas relaciones se necesitan con independencia de su relación con una entidad externa obliga a delimitar de manera más ajustada los compromisos en nuestras adscripciones y, quizás, a formular una buena teoría que arroje luz sobre la relación de implicación, el modo en que ésta determina el contenido conceptual y la forma en que se diferencia de la evidencia al fundamentar nuestros juicios de racionalidad sobre un agente.

Los términos psicológicos usados en las explicaciones agenciales tienen contenido determinado parcialmente por las relaciones que expresan. Estas relaciones no refieren directamente a las consideraciones que el agente considera buenas razones, es decir, no son idénticas a ningún estado de cosas. El contenido de estas expresiones quizás pueda estructurarse a partir de niveles de normatividad de primer orden - propios de una teoría que articule ese contenido inferencialy niveles de segundo orden - relativos, tal vez, a la forma en que deben respetarse principios epistémicos de índole más general, cuando el primer nivel está agotado o no es operativo-. Ambos niveles, en caso de que un día podamos detallarlos, facilitarían la gramática normativa de nuestros términos tradicionales de adscripción. ${ }^{17}$

${ }^{17}$ Este artículo fue redactado en el marco de una investigación financiada por el Ministerio de Educación, Cultura y Deporte adscrita al Departamento de Filosofía de la Universidad de Granada. Agradezco la ayuda de Juan José Acero, director del Departamento durante este periodo. Dos árbitros de Crítica han mejorado el texto sustancialmente con sus comentarios, y me han obligado a eliminar algunas imprecisiones y a reformular algunas partes del argumento. Durante el proceso 


\section{REFERENCES}

Anscombe, G.E.M., 1963, Intention, Cornell University Press, Ithaca. Audi, R., 2001, The Architecture of Reason, Oxford University Press, Nueva York.

Baier, A.C., 1985, "Rhyme as Reason. On Davidson Version of Having a Reason", en E. LePore, B. McLaughlin (comps.), 1985, Actions and Events, Blackwell, Oxford.

Bermúdez, J.L. y A. Millar (comps.), 2002, Reasons and Nature. Essays in the Theory of Rationality, Oxford University Press, Oxford.

Bittner, R., 2001, Doings Things for Reasons, Oxford University Press, Oxford.

Bricke, J., 1996, Mind and Morality. An Examination on Hume's Moral Psychology, Oxford University Press, Nueva York.

Broome, J., 2002, "Practical Reasoning", en Bermúdez y Millar 2002, pp. 85-113.

— 2001, "Normative Practical Reasoning", Proceedings of the Aristotelian Society, vol. supl. 75, pp. 175-193. 419.

Dancy, J., 2003, "Prècis of Practical Reality" y "Replies", Philosophy and Phenomenological Research, vol. 17, no. 2, pp. 423-428 y 468-490.

— , 2000, Practical Reality, Oxford University Press, Oxford.

, 1994, "Why There Is Really No Such Thing As a Theory of Motivation?", Proceedings of the Aristotelian Society, vol. 95, pp. 1-18.

—_, 1993, Moral Reasons, Blackwell, Londres.

(comp.), 2001, Normativity, Blackwell, Londres.

Darwall, S., 2003, "Desires, Reasons, and Causes" Philosophy and Phenomenological Research, vol. 17, no. 2, pp. 436-443.

— 1983 , Impartial Reason, Cornell University Press, Ithaca.

Davidson, D., 1963, "Action, Reasons, and Causes", en Mele 1997, pp. 2742.

Davis, W.A., 2003, "Psychologism and Humeanism", Philosophy and Phenomenological Research, vol. 17, no. 2, pp. 452-459.

Goldman, A., 1988, Epistemology and Cognition, Harvard University Press, Cambridge.

Harman, G., 1986, Change in View, MIT Press, Cambridge, Mass.

—., 1976, "Practical Reasoning", en Mele 1997.

de revisión final, las conversaciones mantenidas con Ruth Chang me ayudaron a comprender mejor el debate entre internistas y externistas normativos, haciéndome ver que la terminología usada en este ámbito está por delimitarse. Lo que sigue, sin embargo, no habría sido posible sin el consejo y el apoyo de María José Frápolli Sanz, que ha leído y mejorado sucesivos borradores de este escrito, animándome a decir algunas cosas de una manera menos oscura. Las imprecisiones y errores que quedan son, como casi siempre, enteramente de quien escribe. 
Hornsby, J., 1997, “Agency and Causal Explanation”, en Mele 1997. , 1980, Actions, Routledge and Kegan Paul, Londres.

Hume, D., 1978, A Treatise of Human Nature, ed. L.A. Selby-Bigge, 2a. ed. rev. por P.H. Nidditch, Oxford University Press, Oxford.

Korsgaard, C.M., 1997, "The Normativity of Practical Reason", en G. Cullity y B. Gaut (comps.), 1997, Ethics and Practical Reasons, Oxford University Press, Oxford.

Mele, A. (comp.), 1997, The Philosophy of Action, Oxford University Press, Oxford.

Millar, A., 2002, "Reasons for Action”, en Bermúdez y Millar 2002, pp. 113135.

Nagel, T., 1986, The View from Nowhere, Clarendon Press, Oxford [versión en castellano: Una visión de ningún lugar, Fondo de Cultura Económica, México, 1996].

- 1970 , The Possibility of Altruism, Princeton University Press, Princeton [versión en castellano: La posibilidad del altruismo, trad. Ariel Dilon, Fondo de Cultura Económica, México, 2004].

Parfit, D., 1997, "Reasons and Motivation", Proceedings of the Aristotelian Society, vol. 71, pp. 99-130.

— 1984, Reasons and Persons, Oxford University Press, Oxford.

Raz, J., 2001, "Explaining Normativity: On Rationality and the Justification of Reasons", en Dancy 2001, pp. 34-59.

Scanlon, T., 1999, What We Owe to Each Other, Harvard University Press, Cambridge.

Schueler, G.F., 2003, Human Rationality and the Teleological Explanation of Action, Oxford University Press, Nueva York.

- 1995, Desire; Its Role in Practical Reason and the Explanation of Action, MIT Press, Cambridge, Mass.

Smith, M., 2004, "Is There a Nexus between Reasons and Rationality?", en S. Tenebaum (comp.), Poznan Studies in the Philosophy of Science and Humanities: New Trends in Moral Psychology, Rodopi, Amsterdam.

— , 2003, "Humeanism, Psychologism, and the Normative Story", Philosophy and Phenomenological Research, vol. 17, no. 2, pp. 460467.

- 1994, The Moral Problem, Blackwell, Oxford.

Wallace, Jay R., 2003, "Explanation, Deliberation, and Reasons", Philosophy and Phenomenological Research, vol. 17, no. 2, pp. 429-435.

Williams, B., 1981, "Internal and External Reasons", reimpreso en B. Williams, 1981, Moral Luck, Cambridge University Press, Cambridge. (Versión en castellano: "Razones internas y externas", La fortuna moral, trad. Susana Marín, Instituto de Investigaciones Filosóficas-UNAM, México, 1993.)

Recibido el 5 de marzo de 2004; revisado el 18 de enero de 2005; aceptado el 9 de marzo de 2005. 